\title{
Letter to the editor: The negative predictive value of preoperative urodynamics for stress urinary incontinence following prolapse surgery
}

\author{
Tsun-Wen Hsiao $^{1,2} \cdot$ Chin-Ru Ker ${ }^{1,2}$ • Cheng-Yu Long ${ }^{1,3}$ (D) \\ Received: 17 March 2019 / Accepted: 16 April 2019 /Published online: 29 April 2019 \\ (C) The International Urogynecological Association 2019
}

To the Editor,

We read with interest the recent article "The negative predictive value of preoperative urodynamics for stress urinary incontinence following prolapse surgery" by Tania Sierra and co-authors [1]. The article addresses the need for concomitant continence surgery with various types of pelvic organ prolapse (POP) surgery. However, we find that the types of POP surgery mentioned in the article are relatively limited because of the absence of transvaginal mesh (TVM) procedures. TVM surgery is clinically effective in repairing POP, despite a safety warning issued by the US Food and Drug Administration (USFDA) in 2017. We found that women had significant prolapse reduction after being treated with single-incision vaginal meshes [2]. A short-term anatomical success rate as high as 98\% can be achieved [2]. The incidence of de novo stress urinary incontinence (SUI) after POP surgery has been reported to be $11 \%$ by Tsia-Shu Lo et al. and $25 \%$ by Kasturi et al. $[3,4]$. The discrepancy in the incidence between these two studies can be explained by the difference of POP surgery where in Kasturi's study only TVM surgery was used [4]. Apparently, the topic of this article by Tania Sierra should be revised because of the absence of TVM surgery in this study. Thus, the negative predictive value in this series may be higher than expected. The possibility of postoperative de novo SUI is an important issue for patients and surgeons. Whether

Cheng-Yu Long

urolong@yahoo.com.tw

1 Graduate Institute of Medicine, College of Medicine, Kaohsiung Medical University, Kaohsiung, Taiwan

2 Department of Obstetrics and Gynecology, Kaohsiung Medical University Hospital, Kaohsiung Medical University, Kaohsiung, Taiwan

3 Department of Obstetrics and Gynecology, Kaohsiung Municipal Hsiao-Kang Hospital, Kaohsiung Medical University,

Kaohsiung, Taiwan and when an anti-incontinence procedure should be done concomitantly with POP surgery remains to be investigated. We are delighted to read this informative article and are looking forward to more studies to be published.

\section{Compliance with ethical standards}

Conflicts of interest None.

\section{References}

1. Sierra T, Sullivan G, Leung K, Flynn M. The negative predictive value of preoperative urodynamics for stress urinary incontinence following prolapse surgery. Int Urogynecol J. 2019. https://doi.org/ 10.1007/s00192-018-03864-y.

2. Ker CR, Lin KL, Loo ZX, Juan YS, Long CY. Comparison of UpholdTM $^{\text {TM }}$ aginal mesh procedure with hysterectomy or uterine preservation for the treatment of pelvic organ prolapse. Sci Rep. 2018;8(1):9438.

3. Lo TS, Bt Karim N, Nawawi EA, Wu PY, Nusee Z. Predictors for de novo stress urinary incontinence following extensive pelvic reconstructive surgery. Int Urogynecol J. 2015;26(9):1313-9.

4. Kasturi S, Diaz SI, McDermott CD, et al. De novo stress urinary incontinence after negative prolapse reduction stress testing for total vaginal mesh procedures: incidence and risk factors. Am J Obstet Gynecol. 2011;205(5):487.e1-4.

Publisher's note Springer Nature remains neutral with regard to jurisdictional claims in published maps and institutional affiliations. 